\title{
An Ocean Model for Climate Variability Studies
}

\author{
K. HASSELMANN \\ Max-Planck-Institut fur Meteorologie, Bundestrasse 55, \\ 2000 Hamburg 13, Federal Republic of Germany
}

\section{Introduction}

The significance of the oceans for climate dynamics has long been recognised and has been repeatedly stressed (for example, in discussions on the development of an international climate research program, cf. GARP Publ. 16, 1975). Nevertheless, comprehensive models of the ocean designed specifically for climate studies are only just beginning to emerge. The slow evolution of ocean climate models does not appear to be due to fundamental modeling problems, but must probably be attributed to the historical development of ocean modeling. For a long time the primary concern of ocean modelers was understanding the mean general circulation of the ocean, rather than studying the time dependent interactions of the ocean within the climatic system. Since the purpose of modeling is largely to devise the most appropriate simplifications for the given class of problems, ocean models designed for studies of the mean general circulation cannot be expected to be optimal also for climate applications.

The first task in constructing an ocean model for climate investigations is therefore to carry out a scale analysis of the ocean system, and its interaction with the atmosphere and other components of the climate system, in order to identify the essential features of the system which need to be properly simulated for the range of space and time scales relevant for climate. Most of the simplifications arising from a restriction to climatic scales have been expressed in various forms in the literature, but in a different context, usually in relation to the mean ocean circulation. It therefore appears useful to review these approaches in the framework of an ocean model designed specifically for time dependent climate variability studies. No attempt is made, however, at a comprehensive or even representative summary of the relevant literature. We refer here to the many excellent published reviews, for example, Bryan (1975, 1979), Pond and Bryan (1976) or Holland (1977, 1979). 
Compared to the atmosphere, the most distinctive features of the oceans are their large heat storage capacity and slow response. These are normally regarded as damping influences, as evidenced, for example, by the smaller seasonal variations of maritime climates as compared with continental climatic regimes. However, the damping of climatic variations through the inertia of the oceans occurs only if the climatic system is forced externally. With respect to internal interactions, the existence of long time constant components gives rise instead to a strong enhancement of the natural lowfrequency variability of the system (Hasselmann, 1976; Lemke, 1977). The mechanism of this amplification is very simple: the natural variability (synoptic scale turbulence) of the atmosphere, which can be regarded as essentially white noise on climatic time scales, is integrated by the slow components of the climatic system and thereby converted into higher variance red noise. It has been shown by Frankignoul and Hasselmann (1977), and Reynolds (1978, 1979) that much of the variance of observed sea surface temperature anomalies can be explained very simply in this manner.

The time scale separation of the atmospheric and oceanic systems greatly simplifies the problem of coupling the two components in an interactive model. Although the inherent short term variability of the atmosphere is important as a stochastic generator of long term climatic variations, there is no need to model the driving short time scale variations of the atmospheric forcing explicitly. The stochastic excitation can be simulated simply by a white noise, random number generator which has the same spatial covariance structure as the atmospheric driving forces (the surface fluxes). The ocean model can then be integrated with a time resolution $\Delta t$ appropriate for climatic variations (of the order 1 month), even though the ocean is in fact driven by stochastic atmospheric forcing terms characterised by internal time scales much smaller than $\Delta t$.

A complete coupled ocean-atmosphere model must, of course, include not only the stochastic atmospheric forcing, but also the mean feedback from the ocean to the atmosphere and the further interaction loop from the atmosphere back to the ocean. As the response time of the atmosphere to changes in boundary conditions is generally short compared with $\Delta t$, the response of the atmosphere to variations of the ocean (sea surface temperature and surface roughness) can be described to a good approximation as a quasiequilibrium stationary adjustment process. If the climate excursions are small, the response can be characterized further by a linear transfer function. It thus appears a reasonable first-order approximation to represent the atmosphere in a coupled oceanatmosphere climate model as a superposition of a white-noise random generator and a closed linear feedback loop, in which variations of the sea surface properties induce variations of the mean atmospheric circulation, which in turn yield changes in the mean (and white noise) atmospheric forcing of the ocean. A simple quasi-stationary atmospheric model of this structure has not yet been implemented in conjunction with an oceanic model. One of the main difficulties lies in the derivation of the linear transfer functions of the 
atmospheric response, which must include the perturbations of the nonlinear eddy fluxes. However, these problems are not our concern here. For our purposes it is sufficient to summarise that, although the short time scale variability of the atmosphere represents an important driving term for the ocean, it has no direct influence on the construction of the ocean model, which need be designed to resolve only climatic time scales greater than about a month.

The limitation to time and space scales relevant for global climatic investigations yields a number of simplifications in the structure of oceanic models, which are discussed in the following sections. The main difference between an oceanic model designed for climate investigations as compared with a standard mean circulation model lies in the emphasis on variability, as opposed to the steady state. Much of the discussion on the role of the oceans in climate has concentrated on the mean oceanic heat transport. It should be noted, however, that the mean heat transport is of interest in itself only for climate variations longer than a few thousand years, for which the ocean circulation can be treated as quasi-stationary. For shorter time scales, variations of the heat transport must be considered in conjunction with the heat storage. Changes in the heat storage, however, affect the density field and the thermohaline circulation, and thereby act back again on the heat transport. Thus variations of the heat and mass transports of the oceans cannot be meaningfully separated. The emphasis in the construction of a climate model of the ocean should therefore not lie solely in the problem of the heat transport, but should concentrate generally on techniques for filtering out the small scale, rapidly varying motions which are irrelevant for climatic time and space scales. In this manner one may hope to derive a thermodynamical model which is sufficiently efficient to be used for systematic investigations of the nonstationary interaction of the oceanic circulation system within the climatic system.

\section{Filtered Equations for the Interior Ocean}

We assume that for global climatic studies the time and horizontal space resolution of the ocean model may be limited in the interior ocean, away from lateral boundaries and excluding a narrow equatorial strip, to 1 month and $500 \mathrm{~km}$, respectively. The one month time resolution corresponds to the generally accepted definition of the lower time scale limit of climatic variability. The spatial resolution of $500 \mathrm{~km}$ may be related through the time resolution to a maximum advection velocity of $500 \mathrm{~km} \mathrm{month}^{-1} \approx 20 \mathrm{~cm} \mathrm{~s}^{-1}$. The rather arbitrary choice of $500 \mathrm{~km}$ is not critical. We shall require in the following mainly that the spatial resolution is large compared with the internal Rossby deformation radius (defined below), which is typically of the order of $50 \mathrm{~km}$.

Under these conditions, the full equations for the ocean can be strongly simplified. Applying the usual hydrostatic and Boussinesque approximations, the complete set of prognostic equations is given by 


$$
\begin{gathered}
\frac{\partial}{\partial t} \underline{u}-f_{\mathcal{u}}+\underline{\nabla} p=q \\
\frac{\partial}{\partial t} \zeta-w_{0}=q_{\zeta} \approx 0 \\
\left.\left.\frac{\partial}{\partial t} \mid \begin{array}{l}
T \\
S
\end{array}\right\}+\mid(\underline{u} \cdot \underline{\nabla})+w \frac{\partial}{\partial z}\right\}\left|\begin{array}{l}
T \\
s
\end{array}\right|=\left|\begin{array}{l}
q_{T} \\
q_{S}
\end{array}\right|
\end{gathered}
$$

where the horizontal velocity $\underline{u}=(u, v)$, surface elevation $\zeta$, salinity $S$ and temperature $T$ define the components of the basic system state vector $\underline{\phi}=(\underline{u}, \zeta, \Upsilon, S) ; \underline{u}=(v,-u)=R \underline{u}$ represents the vector $\underline{u}$ after applying a $90^{\circ}$ right (counter clockwise) rotation $R$ (the notation $\underline{a}_{-}=R \underline{a}$ will be used generally for a rotation $R$ of any horizontal vector $\underline{a}$ ); $f$ is the (latitude dependent) Coriolis parameter; $\nabla=\left\{\left(a \cos ^{\theta}\right)^{-1} \frac{\partial}{\partial \lambda}, a^{-1} \frac{\partial}{\partial \theta}\right\}$ is the gradient operator in spherical coordinates ( $\lambda=$ longitude, $\theta=$ latitude, $a=$ radius of earth) -- we note that the contravarient gradient operator must be distinguished from the covariant divergence operator, $\underline{\nabla}=\left\{(\mathrm{a} \cos \theta)^{-1} \frac{\partial}{\partial \lambda},(\mathrm{a} \cos \theta)^{-1} \frac{\partial}{\partial \theta} \cos \theta\right\}$, appearing later in Eq. (2.5) $w$ is the vertical velocity, with $w_{0}$ the value of $w$ at the surface $z=\zeta ; z$ is the vertical coordinate, taken positive upwards; and $\mathrm{p}$ is the dynamic pressure, defined in terms of a constant reference density $\tilde{p}_{0}$ and pressure deviation $\tilde{p}_{1}$ as $p=\tilde{p}_{1} / \tilde{p}_{0}$ where the total pressure $\tilde{p}=\tilde{p}_{0}+\tilde{p}_{1}$ is represented as the sum of the hydrostatic reference pressure $\tilde{p}_{0}=$ $\mathrm{g} \tilde{\rho}_{\mathrm{O}} \mathrm{z}$ and the pressure deviation $\tilde{\mathrm{P}}_{1}$.

For the large horizontal scales of interest here, $\tilde{P}_{1}$ can be determined hydrostatically from the anomaly $\tilde{\rho}_{1}$ of the density field $\tilde{\rho}=\tilde{\rho}_{0}+\tilde{\rho}_{1}$ relative to the reference density $\tilde{\rho}_{0}$,

$$
p=g \zeta+g \int_{z}^{0} \rho d z
$$

where $\rho=\tilde{\rho}_{1} / \tilde{\rho}_{0}$

The forcing terms on the right-hand side of Eqs. (2.1) and (2.3) represent the sum of turbulent eddy transport terms and, in the case of Eq. (2.1), nonlinear advection (the important advection terms in (2.3) are included explicitly in the left-hand side of the equation). The forcing term on the right-hand side of (2.2) represents the sum of the mass flux due to precipitation or evaporation and the nonlinear slope corrections in the expression for the normal surface velocity. It is generally very small and will be neglected in the following.

The fields $w$ and $p$ represent non-prognostic variables which are related to the components of the state vector $\Phi$ through the diagnostic hydrostatic equation $(2.4)$, the equation of state of sea water, which defines $\rho$ as a function of $T, S$ and $z$ (or, equivalently, the reference pressure $\tilde{\mathrm{P}}_{\mathrm{o}}=\mathrm{g} \tilde{\rho}_{0} z$ ), and the equation of continuity, 


$$
w=-\underline{\nabla} \int_{-h}^{z} \underline{u d} z
$$

where $h(\lambda, \theta)$ denotes the ocean depth.

To close the system of equations, the turbulent mixing terms in the source functions need to be expressed in terms of $\phi$. Furthermore, boundary conditions must be specified. The choice of parameterisation normally affects also the appropriate form of the boundary conditions. However, these questions are not immediately relevant for the discussion of this section and will be considered later.

The system of equations (2.1)-(2.5) contains various classes of solutions characterised by different space and time scales. Thus motions coupled to displacements of the free surface are generally associated with significantly shorter time scales than motions governed only by internal displacements of density surfaces. To separate out these two forms of motion it is customary to divide the system $\Phi_{\sim}$ into a barotropic subsystem $\hat{\phi}=$ $(\underline{\hat{u}}, \zeta)$ and a baroclinic subsystem $\underline{\phi}^{\prime}=\left(\underline{u^{\prime}}, \mathrm{T}, \mathrm{S}\right)$, where the barotropic velocity,

$$
\underline{u}=\frac{1}{h} \int_{-h}^{o} \underline{u d z} \text {, }
$$

is defined as the vertically averaged horizontal velocity and the baroclinic velocity as the residual field $\underline{u}^{\prime}=\underline{u}-\underline{\hat{u}}$.

The associated barotropic and baroclinic components of the vertical velocity,

$$
w=\hat{w}+w^{\prime},
$$

are then defined in terms of the individual continuity relations

$$
\left.\mid \begin{array}{l}
\hat{\mathbf{w}} \\
\mathbf{w}^{\prime}
\end{array}\right\}=-\nabla \int_{-h}^{z}\left\{\left\{\begin{array}{l}
\underline{\hat{u}} \\
\underline{\mathbf{u}^{\prime}}
\end{array}\right\} d z .\right.
$$

Introducing also barotropic and baroclinic components of the pressure field,

$$
p=\hat{p}+p^{\prime}
$$

$\begin{array}{ll}\text { where } & \hat{\mathrm{p}}=\mathrm{g} \zeta \\ \text { and } & \mathrm{p}^{\prime}=\mathrm{g} \int_{\mathrm{z}}^{0} \rho \mathrm{dz},\end{array}$

the system of equations (2.1)-(2.3) can be separated into the two (coupled) subsystems: 


\section{Barotropic system}

where

$$
\begin{gathered}
\frac{\partial}{\partial t} \hat{\hat{u}}-f \underline{\underline{u}}+\underline{\nabla} \hat{p}+\left[\frac{1}{h} \int_{-h}^{0} \nabla p^{\prime} d z\right]=\hat{q} \\
\frac{\partial}{\partial t} \zeta-\hat{w}_{0}=0 \\
\hat{q}=\frac{1}{h} \int_{-h}^{g} g d z ;
\end{gathered}
$$

\section{Baroclinic system}

$$
\begin{aligned}
& \frac{\partial}{\partial t} \underline{u}^{\prime}-f \underline{u}^{\prime}+\underline{\nabla} p^{\prime}-\frac{1}{h} \int_{-h}^{q} \underline{p} p^{\prime} d z=q^{\prime}
\end{aligned}
$$

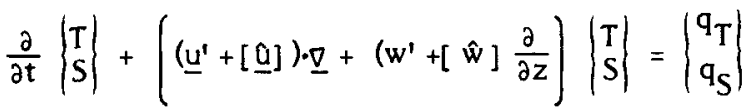

where

$$
q^{\prime}=q-\hat{q}
$$

The interaction between the two systems occurs through the terms in square brackets, the vertically averaged baroclinic pressure gradient in (2.12) and the advection of the density field by the barotropic current in (2.16). (The source functions on the righthand sides normally also provide some coupling.)

Both sets of equations (2.12), (2.13) and (2.15), (2.16) can be simplified for climatic time and space scales. Because of the different inherent time scales, however, the approximations for the barotropic system are different from those for the baroclinic system. The appropriate reductions can be readily derived from a scale analysis of the equations. However, they are perhaps more readily interpreted in terms of the familiar dispersion curves of the linearised systems.

For free, linearised perturbations of a horizontally homogeneous, stably stratified ocean, the terms coupling the two systems can be shown to be negligible. (The baroclinic pressure term in the barotropic momentum equation (2.12), and the vertical barotropic advection term in the density equation (2.16), although linear, are negligible for a horizontally homogeneous basic state for which $\tilde{\rho}_{1}<<\tilde{\rho}_{0}$.) The barotropic and baroclinic equations (2.12), (2.13) and (2.15), (2.16) therefore represent in this case a projection of the full linearised system on to two dynamically distinct subsystems characterised by different sets of normal modes. The dispersion curves for the barotropic and baroclinic modes are shown schematically in Fig. 1 (the computations are based on the $\beta$ plane approximation and a constant stratification, and for the anisotropic Rossby modes only the zonal 
wavenumber dependence is shown).

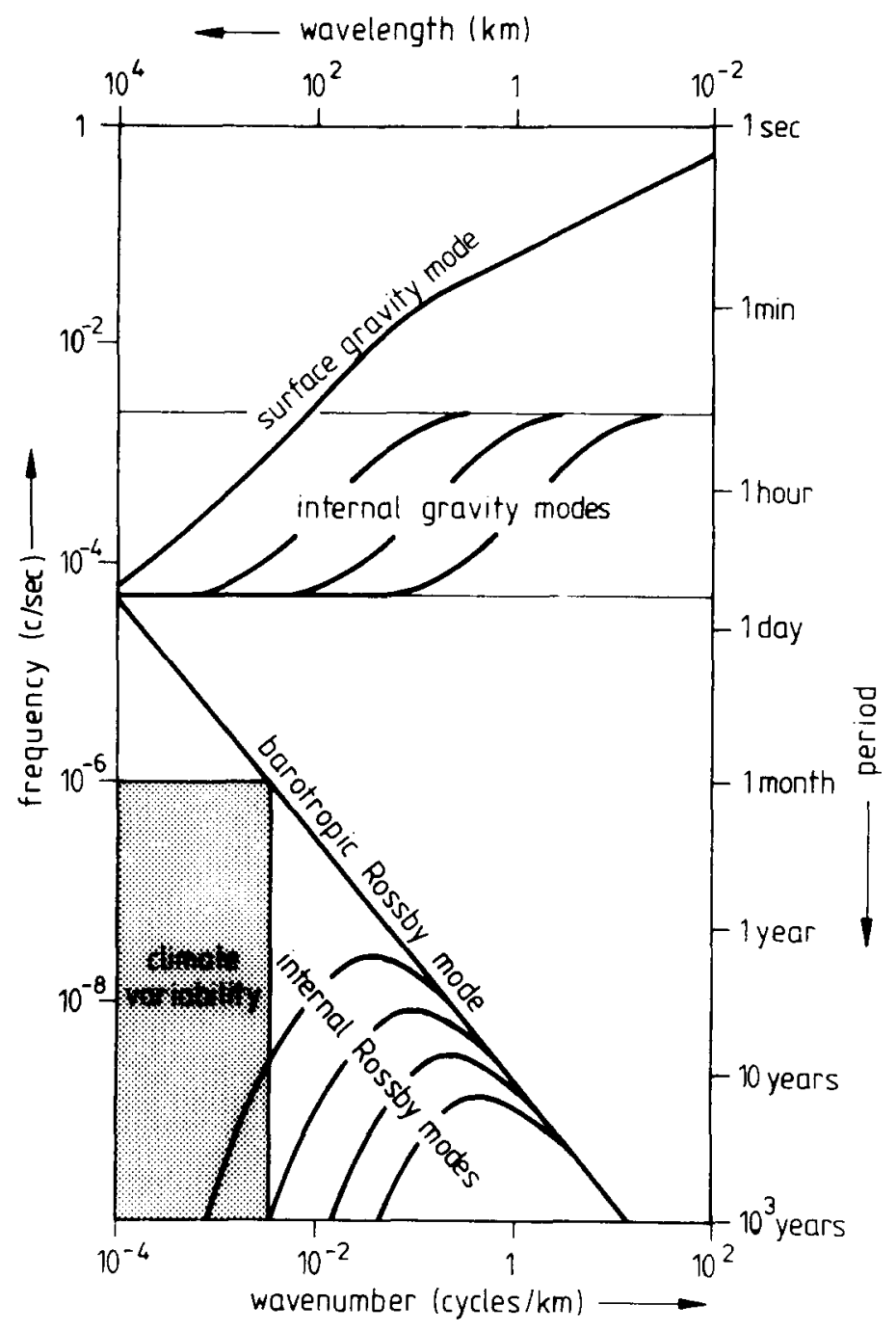

Fig. 1. Dispersion curves for a horizontally homogeneous ocean with density stratification (schematic). The region of interest for climate studies is indicated by the stippled box. 
A characteristic feature of the dispersion curves is the change in slope for wavenumbers near the inverse of the Rossby radius of deformation

$$
R_{d}= \begin{cases}\frac{\sqrt{g h}}{f} & \text { for barotropic modes } \\ \frac{h N}{n f}=R_{\text {int }} & \text { for baroclinic modes, } \\ \text { where } N=g\left(\frac{\partial \rho}{\partial z}\right) \frac{1}{2} \\ \text { is the Brunt-Väisälä frequency and } \\ \text { n denotes the vertical mode number. }\end{cases}
$$

For Rossby waves the wavenumber $R_{d}^{-1}$ marks the transition from the region $k_{x}<$
$R_{d}{ }^{-1}$, in which the waves are governed by a balance between the north-south advection of the planetary vorticity (the $\beta$ effect) and vertical vortex tube stretching, to the region $k_{x}>>R_{d}^{-1}$ of divergence-free waves, in which the $B$ effect is balanced by the time derivative of relative vorticity. The region of time and space scales relevant for climate variations is indicated by the stippled box in Fig. 1 . Since the internal Rossby radius of deformation of the ocean is typically of order $50 \mathrm{~km}$, the baroclinic Rossby waves within the box correspond to the region $k_{x}<R_{d}^{-1}$ in which the time derivative of the vorticity field can be neglected and the motions treated as strictly geostrophic.

The dispersion curves of all other modes are seen to lie well above the climate box; these modes therefore appear only as forced modes at frequencies well below their natural frequencies. In this case the mode response is essentially given by the quasi-stationary zero-frequency response, so that all time derivatives in the equations can be neglected. For barotropic and baroclinic gravity modes the forced response is very small and can be neglected entirely. However, since there is no numerical or analytical advantage in filtering out the (stationary forced) gravity modes we shall retain them together with the more important quasi-stationary forced barotropic Rossby mode. The top right corner of the climate box is seen to reach up to the barotropic Rossby mode dispersion curve. If this limiting high wavenumber, high frequency range of climate variability is important for particular applications, the corresponding region of the barotropic Rossby mode spectrum must be treated prognostically. We shall assume in the following, however, that these effects can be ignored, and that the climate box can in effect be rounded at the corner.

Thus, for the linearised system the time derivatives of both barotropic equations (2.12), (2.13) and the time derivative of the baroclinic momentum equation (2.15) can be neglected, leaving as the only prognostic equation the baroclinic density equation (2.16).

It can readily be shown by direct scale analysis that for climatic time and space scales, which satisfy the inequalities $\frac{\partial}{\partial t}<<f, \underline{\nabla}<\mathrm{R}_{\text {int }}^{-1}$, these conclusions remain valid (with certain exceptions which are discussed in the next section) also for the complete, 
horizontally inhomogeneous, nonlinear system.

We note that although both barotropic and baroclinic velocity fields are determined diagnostically as quasi-stationary equilibrium fields, the governing equations of the two fields are fundamentally different. The baroclinic velocity $\underline{u}^{\prime}$ is determined locally by the gradient of the density field through the (vertically integrated) thermal wind relation (2.15) (for known $q^{\prime}$ ). However, the barotropic velocity $\underline{\hat{u}}$ depends on the unknown barotropic pressure field $\hat{\mathrm{p}}$ in Eq. (2.12) and must be determined globally by the solution of an elliptical partial differential equation (cf. Schulman and Niiler, 1970; Sarkisyan and Ivanov, 1971; Sarkisyan and Keondzhian, 1972). We shall therefore discuss the two systems separately, considering their interaction later in Sections 5 and 6.

\section{The Barotropic System in the Interior Ocean}

The quasi-stationary barotropic velocity field $\underline{\hat{u}}$ is determined by the time independent form of Eqs. (2.12) and (2.13). The solution of these equations for the steady, vertically integrated ocean transport in the presence of a variable bottom topography and density field have been discussed extensively in the literature (cf. Kamenkovich, 1962; Holland, 1967; Welander, 1968; Sarkisyan and Ivanov, 1971; Schulman and Niiler, 1970; Schulman, 1975; and others). The vertically integrated continuity condition (2.13),

$$
w_{0}=-\underline{\nabla} \cdot(h \underline{\hat{u}})=0
$$

is normally used either to eliminate $\underline{\hat{u}}$ from Eq. (2.12), thereby deriving a field equation for $\hat{p}$, or, alternatively, to express $\underline{\hat{u}}$ in terms of a scalar stream function $\psi$,

$$
\underline{\hat{u}}=h^{-1} \nabla \psi,
$$

thereafter eliminating $\hat{p}$. This is normally preferred, as the boundary conditions for $\psi$ are a little simpler that for $\hat{p}$.

Applying the operator $\nabla$ to Eq. (2.12), one obtains the governing equation for $\psi$

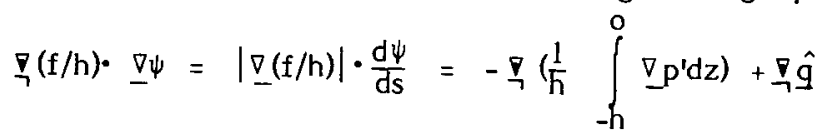

where ds denotes a line increment along a curve $f / h=$ const. (It should be noted in Eqs. (3.2) and (3.3) that, in spherical coordinates, the relations div curl $=0$ and curl grad $=0$ become $\nabla \nabla=0$ and $\underset{\neg}{\nabla}=0$, with two different curl operators $\underset{\neg}{\nabla}$ and $\underset{\neg}{\nabla}$ corresponding to the co- and contragradient operators $\underline{\nabla}, \underline{\nabla}$ )

The baroclinic pressure field acts on the barotropic flow in Eq. (3.3) only through variations of the bottom topography, 


$$
\underset{\gamma}{\nabla}\left(\frac{1}{h} \int_{-h}^{o} \nabla p^{\prime} d z\right)=-\left(\frac{1}{h^{2}} \int_{-h}^{o} \nabla p^{\prime} d z+\frac{1}{h}\left(\underline{\nabla} p^{\prime}\right)_{z}=-h\right) \cdot \underline{\nabla} h .
$$

The order of the differential equation (3.3), and therefore also the required form of the boundary conditions, depends on the form assumed for the barotropic forcing $\hat{q}$. In the interior ocean the (grid scale) nonlinear momentum advection terms are negligible, so that $g$ is determined by the (sub scale) turbulent momentum fluxes. Assuming the usual anisotropic turbulent friction expression,

$$
g=v_{V} \frac{\partial^{2} \underline{u}}{\partial z^{2}}+\nu_{H} \Delta \underline{u}
$$

where $\Delta=\underline{\nabla} \cdot \underline{\nabla}$ denotes the Lapacian operator and $\nu_{\mathrm{V}},{ }_{\mathrm{H}}$ represent vertical and horizontal eddy viscosity coefficients, one finds

$$
\underline{\nabla} \tilde{q} \approx \underline{\nabla}\left(\frac{\underline{\tau}}{h}\right)-\frac{1}{h} \sqrt{\frac{f}{2} v_{v}} \Delta \psi+\frac{\nu_{H}}{h} \Delta^{2} \psi+L\left(\underline{u^{\prime}}\right)
$$

where $I$ is the surface (wind) stress and $L$ is a linear operator acting on the baroclinic velocity field $\underline{\underline{u}}$. The exact form of $L$ is irrelevant for the following; it derives from friction terms associated with the baroclinic velocity analogous to the barotropic contributions in the second and third terms on the right-hand side of (3.6) and contributes to the barotropic forcing because the integral of the forces over the water column is in general non-zero.

The expression for the vertical friction term in (3.6) applies for a deep ocean in which the vertical friction stresses are confined to relatively thin, separated Ekman layers at the top and bottom of the ocean. Both vertical and horizontal friction terms have furthermore been simplified by ignoring terms arising from the spatial variability of $h$ and $f$. Although this cannot be strictly justified by scale considerations, the approximation is adequate for our purposes and appears physically compatible with the basic arbitrariness of the turbulent closure assumptions.

The horizontal and bottom friction terms represent the highest spatial derivatives in (3.6) and therefore determine the structure of the differential equation (3.3) and the boundary conditions of the problem. However, estimates of the orders of magnitude of these terms for friction coefficients $v_{V}, v_{H}$ typically used in ocean models show that they are generally negligible in the interior of the ocean. The higher derivatives become important only in regions of high gradients near lateral boundaries and the equator - i.e. in regions which have been specifically excluded from the present considerations. In the interior ocean the $\psi$-equation therefore reduces to the first-order characteristic equation 


$$
|\underline{\nabla}(\mathrm{f} / \mathrm{h})| \cdot \frac{\mathrm{d} \psi}{\mathrm{ds}}=-\nabla\left(\frac{1}{h} \int_{-h}^{0} \nabla p^{\prime} \mathrm{dz}\right)+\nabla\left(\frac{\tau}{\bar{h}}\right)=\mathrm{Q}
$$

Equation (3.7) represents a generalised Sverdrup relation, in which the $\beta$ effect is replaced in the left-hand side by a combined $\beta$-bottom topography term, and the net forcing torque $Q$ includes the curl of the vertically averaged baroclinic pressure gradient in addition to the curl of the wind stress.

The boundary condition required for (3.7) is that $\psi$ must be prescribed at one point on each of the characteristic curves $\mathrm{f} / \mathrm{h}=$ const. Physically, the condition of no flux through the edges of the ocean yields the boundary condition $\psi=$ const. along all coasts. If a curve $\mathrm{f} / \mathrm{h}=$ const. intersects two different coasts, as is normally the case, on the west and east sides of the ocean basins, only one of the two boundary values may be used as boundary condition for (3.7). It is well known, from considerations of the irreversibility and stability of the complete equation including friction terms - or, alternatively, from the asymptotic solution of the time dependent case - that the characteristic equation must be integrated from east to west, or, more generally, for arbitrary topography, with the higher values of $\mathrm{f} / \mathrm{h}$ to the right relative to the direction of integration. Thus the east coast boundary value must be used as the initial condition.

To satisfy the second boundary condition a narrow boundary layer must be inserted at the western coast in which the full equation (3.3), including the higher derivatives in Eq. (3.6), is solved. If horizontal friction is included, the additional non-slip boundary conditions require boundary layers at both coasts. However, it can be shown that to first order the eastern boundary layer does not affect the eastern boundary value $\psi$ required for the solution of the interior regime.

The contours of constant $f / h$ for the global ocean are shown in Fig. 2 (by courtesy of E. Maier-Reimer). All contours either originate and terminate at coasts, or represent closed curves. If $h$ approaches zero continuously as one approaches a coast, the points of the characteristics must lie on intersections of the coastlines with the equator. However, the contours tend to become closely concentrated along the coasts, so that if boundary layers are taken into account, the points at which the contours cross from the interior region into the lateral boundary layers occur effectively at finite latitudes.

\section{Closed $\mathrm{f} / \mathrm{h}$ Contours}

Closed $f / h$ isolines which lie entirely within the interior ocean, without entering a lateral boundary layer or the equatorial region, occur in several regions of the ocean. They can arise through the presence of islands (in particular Antarctica), but also through sea mounts or ridges, such as the mid-Atlantic ridge. In these regions the quasi-stationary 

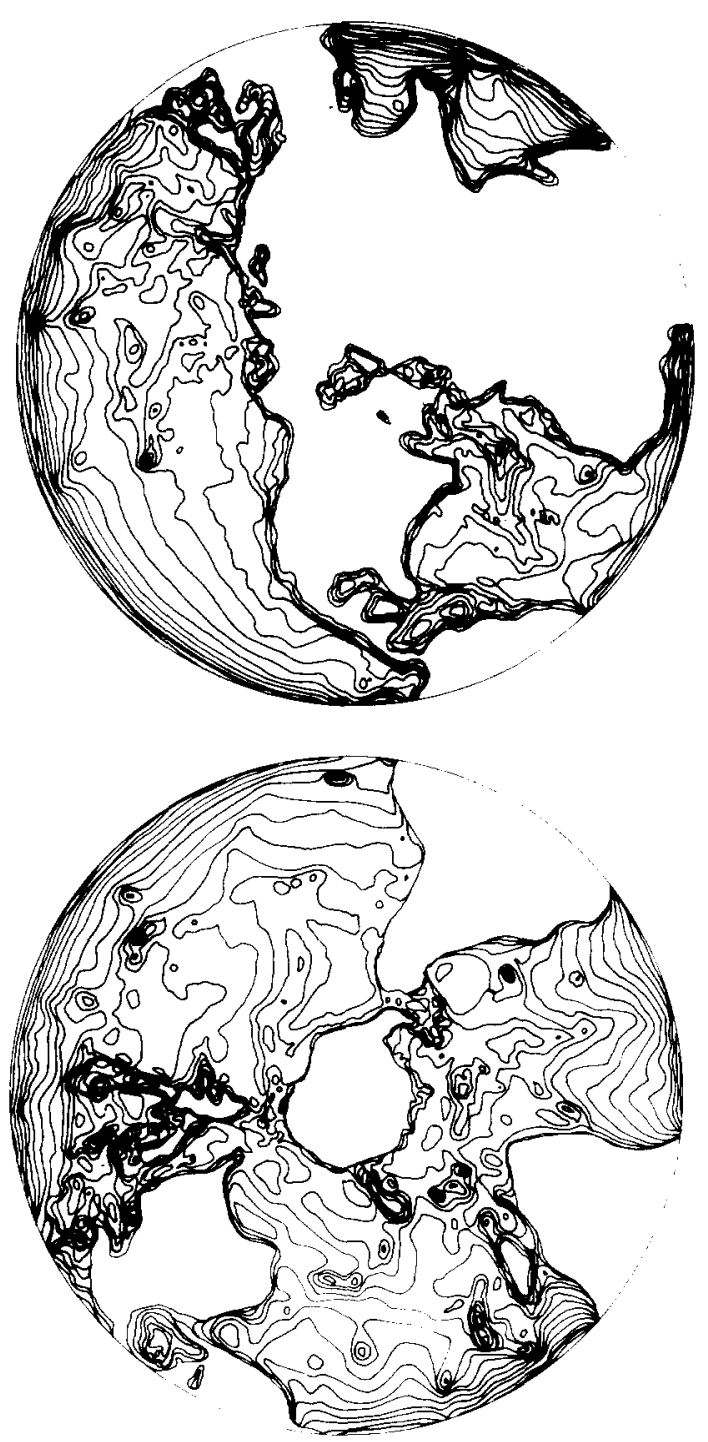

Fig. 2. Contours of constant $\mathrm{f} / \mathrm{h}$ in northern (top) and southern (bottom) hemisphere projections centered on the poles. All oceans contain a number of (generally localised) regions with closed $f / h$ contours. 
approximation (3.7) for the barotropic flow breaks down. Integrating $\frac{\mathrm{d} \psi}{\mathrm{ds}}$ around a closed $\mathrm{f} / \mathrm{h}$ contour, one obtains from Eq. (3.7) the condition

$$
\oint \frac{d \psi}{d s} d s=\oint\left\{-\nabla\left(\frac{1}{h} \int_{-h}^{0} \nabla p^{\prime} d z\right)+\left.\nabla \cdot\left(\frac{\tau}{h}\right)|| \underline{\nabla}(f / h)\right|^{-1} d s=0 .\right.
$$

Since the density field, as a prognostic field, and the wind stress may be arbitrarily specified, there is no reason to assume that Eq. (3.8) will in general be satisfied, and it must be concluded instead that the original equation (3.7) is inconsistent. The origin of the inconsistency is readily recognised: for vanishing forcing, the left-hand side of Eq. (3.7) reduces to the well-known theorem that the general solution for barotropic quasigeostrophic flow over variable topography is given by an arbitrary non-divergent flow following contours of constant $\mathrm{f} / \mathrm{h}$ (potential vorticity). If such a flow is now exposed to a non-gradient force field characterised by a net torque around closed streamlines, the flow will be accelerated. A steady solution can exist only if balancing friction forces are considered.

Since the frictional forces in the interior ocean are small, the balance will normally require a long time to become established, and it can therefore no longer be argued that the barotropic flow achieves a quasi-equilibrium state in a time short compared with the resolution time of the model. In terms of the dispersion curve discussion of the previous section, the quasi-equilibrium treatment of the barotropic modes was justified by the fact that, for a constant depth ocean, the frequencies of the barotropic modes were large compared with the inverse of the model resolution time. However, if variable topography with closed $\mathrm{f} / \mathrm{h}$ contours is considered, zero frequency trapped barotropic Rossby modes are seen to exist which no longer satisfy this inequality. (Very low frequency barotropic modes are also found in a constant depth ocean if non-zonal wave numbers, not depicted in Fig. 1, are considered. However, the stationary approximation remains valid, as the group velocity remains large. The essential feature which leads to a breakdown of Eq. (3.7) is the trapping of vorticity along closed contours.)

It has often been conjectured (e.g. Welander, 1968) that in the mean ocean circulation the wind stress torque acting around closed $\mathrm{f} / \mathrm{h}$ contours is actually largely balanced by the baroclinic pressure term, so that Eq. (3.8) is in fact approximately valid. Physically, this occurs through a compensation of the barotropic pressure at depth by the baroclinic pressure field, thereby essentially decoupling the circulation from the bottom topography. The details of this compensation mechanism and the degree of compensation itself are not well understood, but the adjustment times involved must at all events lie in the range of the baroclinic response time, so that an equilibrium cannot be assumed in climate variability studies addressed to the same time scale range.

However, a simple formulation of the time dependent barotropic equations for closed $\mathrm{f} / \mathrm{h}$-isolines can be readily derived. The solution may be divided into a time dependent 
component representing the resonant excitation of a trapped potential vorticity gyre by the mean torque averaged over a closed contour, and a quasi-stationary response to the residual vorticity force field, after subtraction of the mean torque.

The time dependent barotropic vorticity equation follows from (2.12), (2.13),

$$
\frac{\partial}{\partial t}\left\{-\underline{\nabla}\left(\frac{1}{h} \underline{\nabla} \psi\right)-\frac{f}{h} \zeta\right\}+\left(\underline{\nabla} \frac{f}{h}\right) \cdot \underline{\nabla} \psi=Q
$$

where the stream function and surface displacement are related in the quasi-geostrophic approximation through

$$
\frac{f}{h} \underline{\nabla} \psi=-g \underline{\nabla} \zeta-\frac{1}{h} \int_{-h}^{o} \underline{\nabla} p^{\prime} d z+\hat{g} .
$$

If the torque is separated into two terms,

$$
\mathrm{Q}=\overline{\mathrm{Q}}+\mathrm{Q}^{\prime}
$$

where

$$
\overline{\mathrm{Q}}=\overline{\mathrm{Q}}(\mathrm{f} / \mathrm{h})=\left\{\boldsymbol{\phi}|\underline{\nabla}(\mathrm{f} / \mathrm{h})|^{-1} \mathrm{ds}\right\}^{-1} \oint|\underline{\nabla}(\mathrm{f} / \mathrm{h})|^{-1} \mathrm{Qds}
$$

represents a weighted mean torque over a closed $f / h$ isoline and $Q^{\prime}$ is the residual torque, the solution of (3.10), (3.11) can be expressed as the sum $\psi=\overline{\bar{\psi}}+\psi^{\prime}$ of a non-stationary gyre stream function $\bar{\psi}=\bar{\psi}(\mathrm{f} / \mathrm{h}) \equiv \bar{\psi}(\alpha)$ which is constant along contours $\mathrm{f} / \mathrm{h} \equiv \alpha(\underline{\mathrm{x}})=$ const. and a quasi-stationary residual $\psi^{\prime}$.

The equation for $\bar{\psi}$ follows by forming the weighted average of (3.9) over a closed $\mathrm{f} / \mathrm{h}=\alpha$ isoline,

$$
\frac{\partial}{\partial t}\left\{\frac{\partial \bar{\psi}}{\partial \alpha} \overline{\left[h^{-2} \cdot \underline{\nabla} h \cdot \underline{\nabla} \alpha-h^{-1} \Delta_{\alpha}\right]}-\frac{\partial^{2} \bar{\psi}}{\partial \alpha^{2}} \overline{\left[h^{-1} \underline{\nabla} \alpha \cdot \underline{\nabla}_{\alpha}\right]}-\alpha \bar{\zeta}\right\}=Q .
$$

The relation between $\bar{\psi}$ and the weighted mean displacement $\bar{\zeta}$ follows by taking the weighted mean of Eq. (3.10), and noting that the mean forcing $\bar{Q}$ is small in the balance equation for a resonantly generated trapped vorticity gyre flow which is integrated over a time large compared with $f^{-1}$,

$$
\alpha \frac{\partial \bar{\psi}}{\partial \alpha}=-g \frac{\partial \bar{\zeta}}{\partial \alpha}
$$

The equation for $\psi^{\prime}$ is given by the difference of Eqs. (3.9) and (3.10) and yields to first order, for $\frac{\partial}{\partial t} \psi<<\psi$, the time independent vorticity equation

$$
\left|\underline{\nabla}\left(\frac{f}{h}\right)\right| \frac{d \psi^{\prime}}{d s}=Q^{\prime} \text {. }
$$

In Eq. (3.15) the friction terms in ' $Q^{\prime}$ can be neglected, as elsewhere in the interior ocean. It is not known, however, whether friction can be neglected also to first order in the time dependent equation (3.13) for the trapped vorticity gyre, the steady state being 
achieved in this case primarily by the baroclinic adjustment process, or whether the frictional torque represents an essential term in the final steady state balance.

Independent of this question, however, it may be concluded that in regions of closed $\mathrm{f} / \mathrm{h}$ isolines in the interior ocean the stationary barotropic $\psi$-equation (3.7) applies only for the residual stream function $\psi^{\prime}$ driven by the residual torque field $Q^{\prime}$, whereas the mean torque $\bar{Q}$ around an isoline generates a non-stationary trapped vorticity gyre $\bar{\psi}$ in accordance with Eqs. (3.11), (3.12). The field $\bar{\psi}$ must therefore be included together with the fields $T$ and $S$ as a component of the prognostic system.

A general discussion of the response properties of the system $(\bar{\psi}, \mathrm{T}, \mathrm{S})$ to external forcing on various time and space scales typical for climate variations does not appear to have been attempted. As a simple example the response of the system $(\bar{\psi}, \mathrm{T}, \mathrm{S})$ to seasonal forcing is considered briefly in Section 6.

The question of closed $\mathrm{f} / \mathrm{h}$ contours has some bearing on the boundary value problem for islands. The boundary condition of zero normal mass flux along coasts requires $\psi=$ const., but does not specify the constant. Since $\psi$ is only defined to within a constant, this presents no difficulty if the ocean is bounded by a single connected coastline. For the global ocean, however, this is not the case. The relation between the constant $\psi$ values for different, disconnected coasts ("islands") can be established by noting that the $\psi$-equation (3.3) was originally obtained by taking the curl of (2.12) to eliminate $\nabla \hat{p}$. Equation (2.12) can be recovered from (3.3) by integration, but in place of the gradient field $\nabla \hat{p}$ one obtains then as general integration constant a vector field a with the property $\underline{\nabla} \underline{\underline{a}}=0$. For a simply connected region a may be immediately identified with $\underline{\nabla} \hat{p}$ through the definition

$$
\hat{p}(\underline{x})=\int_{x_{0}}^{x} \text { ad } \underline{x},
$$

where the integration curve may be chosen arbitrarily. For a multiply connected region, however, a may be identified with a gradient field in this manner only if the condition $\oint_{\mathbf{a d x}}=0$ is satisfied for all closed curves encircling islands, for which Stokes' theorem cannot be applied. From (2.12) one obtains then for $\psi$ the additional condition (cf. McWilliams, 1977; Pedlosky, 1979)

$$
\oint\left\{\frac{1}{h} \frac{\partial}{\partial t} \underline{\nabla} \psi+\frac{f}{h} \underline{\nabla} \psi+\frac{1}{h} \int_{-h}^{0} \underline{\nabla} p^{\prime} d z-\hat{g}\right\} d \underline{x}=0 .
$$

The time dependent acceleration term has been included in Eq. (3.16), but it is assumed that the quasi-geostrophic, non-divergent approximation, with $\underline{\hat{u}}$ given by the stream function $\psi$ in accordance with Eq. (3.2), can be retained.

The closed integration contour can be chosen to follow close to, but not exactly along, the coast line, so that the curve lies just outside the coastal boundary layer and still within the interior, linear, frictionless region of the ocean. On sections of the coast corresponding to terminal points of $\mathrm{f} / \mathrm{h}$ contours which represent "upstream" initial points 
of the characteristics $f / h=$ const., $\psi$ is constant. On these sections the second term in the integral vanishes, since $\underline{\nabla} \psi d \underline{x}=0$. Along sections of the coast corresponding to "downstream" terminal points of $\mathrm{f} / \mathrm{h}$ isolines, however, $\psi$ cannot be prescribed, and the second integrand is in general nonzero.

The method for determining the unknown constant $\psi_{\text {up }}$ on the "upstream" section of the coast depends on the topology of the $f / h$ contours. If the island is not encircled by a closed $f / h$ isoline, there must exist characteristics which leave the island without returning back again, terminating at some other, disconnected coast. Conversely, there exist also characteristics from disconnected coasts which terminate on "downstream" coast sections of the island. In this case, the stationary form of Eq. (3.16) can be used. The stream function $\psi$ changes discontinuously at the transition points between the sections of the coast containing the "downstream" terminal points of characteristics from other coastlines and the remaining coastal sections containing "upstream" points or "downstream" points of characteristics originating from the same coast. The integral of the term $f / h \underline{\nabla} \psi d \underline{x}$ across such a discontinuity yields $f / h$ (discontinuity in $\psi$ ), and Eq. (3.16) then yields a simple relation to determine the unknown constant $\psi_{\text {up }}$.

If, on the other hand, the island is completely encircled by a closed $\mathrm{f} / \mathrm{h}$ isoline, the time dependent form of Eq. (3.16) should be used. However, the problem can be related very simply in this case to the solution of the time dependent equations (3.13)-(3.15) for the trapped vorticity gyre. The smallest closed $\mathrm{f} / \mathrm{h}$ contour which encircles the island must be tangent to the (smaller) closed integration curve in (3.16) at the nearest point of the $\mathrm{f} / \mathrm{h}$ isoline to the island. The value of $\psi$ of this smallest $\mathrm{f} / \mathrm{h}$ isoline, as determined from the trapped vorticity gyre equations, therefore also determines the constant $\psi$ up along the "upstream" section of the island coastline.

\section{The Baroclinic System in the Interior Ocean}

It is convenient to divide the baroclinic interior ocean (excluding boundary currents) into a seasonal boundary layer, consisting of the surface mixed layer and the seasonal thermocline, and the abyssal ocean, extending from beneath the seasonal boundary layer through the main thermocline to the ocean bottom. There is some evidence (Stommel and Schott, 1977; Schott and Stommel, 1978; Wunsch, 1978) that in large regions of the abyssal ocean the eddy transport of momentum, heat and salinity may be negligible. The prognostic equations for $\mathrm{T}$ and $\mathrm{S}$ then reduce to the pure advection equations. The baroclinic velocity can be determined geostrophically from the density field, with an additional depth independent correction term which arises through the subtraction of the barotropic contribution of the surface Ekman transport. Applying a rotation $\mathrm{R}$ to the time independent form of Eq. (2.15) and substituting $\hat{g}=\frac{T}{h}$, one obtains 


$$
\underline{u}^{\prime}=\frac{1}{\mathrm{f}}\left\{-\underset{\tau}{ } \mathrm{p}^{\prime}+\frac{1}{\mathrm{~h}} \int_{-\mathrm{h}}^{\mathrm{o}} \nabla \mathrm{p}^{\prime} \mathrm{dz}-\frac{\frac{\tau}{\mathrm{T}}}{\mathrm{h}}\right\}
$$

The integration of the advective density equations (2.16) for the abyssal ocean is mathematically straightforward, once $\underline{u}^{\prime}, w^{\prime}$ have been determined by (4.1), (2.8) and the barotropic velocities $\hat{\hat{u}}, \hat{w}$ by the diagnostic or diagnostic-prognostic system of equations discussed in the previous section. As boundary conditions $T$ and $S$ must be specified on all boundary surfaces with inflow; on the outflow boundary surfaces $\mathrm{T}$ and $\mathrm{S}$ are determined internally by the integration of the advection equations.

If stationary solutions are sought, the adiabatic density advection equations cause problems whenever closed streamlines occur within the interior abyssal ocean. In this case the initial density values on the closed streamlines are maintained indefinitely, and the asymptotic equilibrium solution is not determined uniquely by the boundary conditions. However, this problem does not arise in time dependent variability studies, although it may be physically more realistic to include also in this case a weak diffusion in $\mathrm{S}$ and $\mathrm{T}$ when very long term variations are considered.

Another cause for a possible breakdown of the adiabatic density advection equations is the development of hydrostatically unstable density profiles. In this case some form of convective adjustment parameterisation must be included. However, regions of the abyssal ocean circulation which tend to develop hydrostatically unstable stratifications through density advection are not known.

Although the concept of adiabatic density advection in the abyssal ocean has been tested by local diagnostic relations such as the B-spiral (Stommel and Schott, 1977; Schott and Stommel, 1978) and yields reasonable water mass transport properties (Sverdrup et al., 1942; Wunsch, 1978), the approach has not yet been systematically tested in ocean variability studies. A complete investigation of this question requires the coupling of the adiabatic abyssal circulation to the diabatic boundaries of the interior abyssal region, namely the surface seasonal layer, the lateral boundary layers and the equatorial region. However, certain aspects of the time variability of the abyssal ocean can be studied without considering the complete system, as will be discussed in Section 6 .

The description of the seasonal layer has been the subject of numerous investigations (cf. Kraus, 1977) and will not be reviewed here. The matching of the seasonal layer to the abyssal ocean presents no basic difficulty, since the appropriate boundary conditions for each system, the prescription of $T$ and $S$ on boundaries with inflow, are mutually complementary.

\section{The Lateral Boundary Layers and Equatorial Region}

The equations for the interior ocean break down along the lateral boundaries because 
the horizontal gradients and velocities become so large that frictional and nonlinear terms become important. Also, the horizontal scales can no longer be regarded as large compared with the internal Rossby radius of deformation. The same conditions apply along the equator, where the geostrophic constraint vanishes and the internal Rossby radius becomes infinite.

For a rigorous treatment of these regions a fully nonlinear time dependent model based on the primitive equations, or at least, away from the equator, on the unmodified nonlinear quasi-geostrophic equations, is needed. A complete ocean model consisting of a high resolution, nonlinear, time dependent description of the boundary and equatorial regions and a simplified density-advection treatment of the interior ocean has not yet been constructed. It can be questioned, however, whether this is in fact necessary. The boundary and equatorial regions probably represent strongly diabatic regions, in which mixing by sub-scale quasi-geostrophic eddies or smaller scale turbulence represent important processes. As long as these turbulent processes are not fully understood, it can be argued that a detailed, high resolution model of these regions cannot be more reliable than the parameterisation of the governing processes, and that a much simpler bulk parametrical description of the current systems in the spirit of mixed layer models, or the classical integrated boundary layer models of aerodynamics, may serve equally well. Techniques for projecting the full set of prognostic equations of a continuous system on to a finite set of prognostic equations for a small number of discrete parameters used in an approximate "bulk" description of the system have been developed and applied successfully in other fields (e.g. for the transport equation of surface wave spectra, cf. Hasselmann et al., 1973; Günther et al., 1979). However, this approach has not yet been exploited in ocean modeling. Rather, simple bulk models of the return flow in western boundary currents, coupled to an abyssal density advection and bulk seasonal layer model in the interior ocean, could well shed light on a number of open first-order questions regarding the response of the ocean to forcing by variable surface heat fluxes and stresses on different space and time scales.

\section{Seasonal Variability of the Interior Oceanic Circulation}

As an example of the application of a simplified oceanic circulation model to climate variability studies, we consider the variability of the oceanic circulation and heat transport on seasonal time scales. The annual cycle of the ocean is of considerable interest as the largest and most easily measurable signal of oceanic variability. A better understanding of the annual cycle has therefore played a prominent role in recent proposals to initiate a pilot long term ocean monitoring program for studying the variability of the interior ocean and ocean boundary current systems.

The problem of seasonal variations of the ocean, both in the interior ocean and the near surface seasonal layer (the mixed layer plus seasonal thermocline) has been considered 
by several authors. Perhaps the most comprehensive discussion, supported by detailed numerical calculations, is given in Gill and Niiler (1973). Here we attempt only to summarise some of the principal features as an illustration of the model properties described in the previous sections. Since we have not attempted to specify models for the lateral boundary currents and equatorial regions, we shall limit our discussion to the interior ocean, considering the boundary and equatorial regions only to the extent that they influence the interior region (the influence is small).

The seasonal time scale lies between the characteristic time scales for the adjustment of the barotropic and baroclinic velocity fields and density changes by advection. In this time scale range the separation of the interior ocean circulation into a quasi-equilibrium barotropic system and a more slowly varying baroclinic system proves particularly useful.

We assume, as in Section 4, that diabatic variations of temperature and salinity (which we refer to in the following simply as density) are restricted to the near surface seasonal layer. Below this layer local changes in density are assumed to occur only through advection. The structure of the velocity field responsible for the mass and heat transport differs significantly depending on whether one is considering a region of open or closed $\mathrm{f} / \mathrm{h}$ contours, and we therefore discuss these two regimes separately.

\section{Open $f / h$ Contours}

In these regions, which cover most of the global ocean, the barotropic and baroclinic velocity fields can be computed as quasi-stationary equilibrium fields in accordance with Eqs. (3.7) and (4.1). Assuming that the seasonal density variations $p_{s}$ below the seasonal layer are small (we shall show a posteori that this is indeed the case), the seasonal baroclinic velocity $\underline{u}_{s}^{\prime}$, and therefore the net velocity $\underline{u}_{S}=\underline{\underline{u}}_{S}+\underline{u}_{s}^{\prime}$, are essentially independent of depth in the abyssal ocean. In the seasonal layer itself the velocity field $u_{s}^{\prime}$ consists of the local Ekman velocity $\underline{u}_{e}$, the local baroclinic geostrophic velocity $u_{g}$ generated by horizontal gradients of the density variations $\rho_{s}$ in the seasonal layer, and the nonlocal barotropic velocity $\hat{\mathrm{u}}_{\mathrm{x}}$, which is determined mainly by the curl of the seasonal variation $\frac{\tau}{S}$ of the wind stress.

The orders of magnitude of these velocities averaged over the depth $\mathrm{H}$ of the seasonal layer can be estimated as

$$
\begin{aligned}
& u_{e}=O\left(\tau_{s} / f H\right) \\
& u_{g}=O\left(g \rho_{s} H / f L\right) \\
& \hat{u}_{s}=O\left(\tau_{s} / B L H\right)
\end{aligned}
$$

where $L$ represents a typical horizontal scale of the seasonal layer (assumed for simplicity to be of the same order as the horizontal scale of $\tau_{s}$ ) and $B=\frac{1}{a} \frac{d f}{d \theta}$ is taken as 
representative of the order of magnitude of $|\underline{\nabla}(\mathrm{f} / \mathrm{h})| \cdot h$. Below the seasonal layer, $u_{g}$ and $\hat{u}_{s}$ have the same values as in the seasonal layer, but the Ekman contribution becomes $u_{e d}=$ $u_{e} \mathrm{H} / \mathrm{h}=O\left(\tau_{s} / \mathrm{fh}\right)$. (Note that although the Ekman velocity itself vanishes below the Ekman layer, a contribution to the baroclinic velocity at depth nevertheless remains through the formal separation of $\underline{u}$ into a barotropic component and a baroclinic component $\underline{u}$ ' with vanishing vertically integrated transport; cf. Eq. (4.1)).

Taking typical mid-latitude values $\tau_{\mathrm{s}}=10^{-4} \mathrm{~m}^{2} \mathrm{~s}, \mathrm{H}=50 \mathrm{~m}, \mathrm{~h}=4 \times 10^{3} \mathrm{~m}, \mathrm{f}=$ $10^{-4} \mathrm{~s}-1, L=2 \times 10^{6} \mathrm{~m}, \beta=10^{-11} \mathrm{~m}^{-1} \mathrm{~s}^{-1}, \rho_{\mathrm{s}}=10^{-3}$, one obtains $u_{\mathrm{e}} \approx 0.02 \mathrm{~m} \mathrm{~s}^{-1}, \mathrm{u}_{\mathrm{g}} \approx$ $2 \times 10^{-3} \mathrm{~m} \mathrm{~s}^{-1}, \hat{u}_{\mathrm{s}} \approx 10^{-3} \mathrm{~m} \mathrm{~s}^{-1}$ and $u_{\mathrm{ed}} \approx 2 \times 10^{-4} \mathrm{~m} \mathrm{~s}^{-1}$.

Using the estimates of the velocities below the seasonal layer, one can estimate now also the changes in density $\rho_{\text {sd }}$ induced by advection in the deep ocean, and the associated seasonal modulation $u_{g d}$ of the abyssal baroclinic velocity. The advective vertical displacements $\Delta z$ are of the order of the seasonal Ekman pumping velocity $\tau_{s} / \mathrm{fL}$ times the duration of a season, say, $\mathrm{T}_{\mathrm{s}}=100$ days $\approx 10^{7} \mathrm{~s}$. This yields $\Delta z \approx 5 \mathrm{~m}$. The horizontal displacements are of the order $\Delta x=\left(\hat{u}_{s}+u_{g}\right) T_{s} \approx 30 \mathrm{~km}$. These values correspond to density changes in the main thermocline in the range $\rho_{s d} \approx 10^{-5}$. The associated changes of the deep baroclinic velocity are similarly negligible, of order $10^{-4} \mathrm{~m} \mathrm{~s}^{-1}$. Thus on a seasonal time scale, the ocean beneath the seasonal layer responds independently of depth only to the pressure loading induced by changes in the seasonal layer stratification and the wind forcing.

The small horizontal advection of the density field justifies a posteori also the neglect of boundary layer back interactions on the seasonal time scale, as these can affect the interior ocean only through the advection of density from the boundaries into the interior.

The implication of these seasonal velocity variations for the annual heat transport cycle cannot be discussed solely in terms of the amplitudes of the seasonal wind forcing $\tau_{s}$ and seasonal layer density (temperature) variations $\rho_{s}\left(T_{s}\right)$, since the seasonal heat transport depends, through the nonlinearity of the advective terms, on the horizontal and vertical structure of the mean temperature distributions relative to the structure of the velocity variations. In general it can be estimated that the seasonal variations of heat flux are greater in the seasonal layer than in the deeper ocean. This is largely due to the fact that the seasonal component of the abyssal mass transport - in contrast to the mean circulation - is not compensated below the main thermocline. Thus most of the mass transport occurs at depths in which the temperatures are fairly uniform and the heat transport is relatively small.

The seasonal variations of the oceanic heat flux are generally comparable with the mean heat flux, as inferred also by Oort and Vonder Haar (1976), and Ellis et al. (1978) from satellite and meteorological observations. Qualitatively similar results were simulated by Bryan and Lewis (1979) in numeral experiments using a high resolution three dimensional 
ocean general circulation model. It appears, however, that the principal features of the seasonal variations of the circulation and heat flux in the interior ocean can be determined more simply (in regions of the ocean corresponding to open $f / h$ isolines) using seasonal layer models, augmented by straightforward diagnostic calculations of the barotropic velocity field in accordance with the generalised Sverdrup relation (3.7).

\section{Closed $\mathrm{f} / \mathrm{h}$ Contours}

In regions of closed $\mathrm{f} / \mathrm{h}$ contours, the barotropic field can no longer be treated as a quasi-stationary equilibrium field, but evolves slowly in time in accordance with the nonstationary gyre equation (3.11). To estimate the order of magnitude of the barotropic seasonal response in these regions, we assume for simplicity that the seasonal variations $Q_{s}$ of the mean torque $\bar{Q}$ around a closed $f / h$ contour are sinusoidal with period $T_{a}=1$ year. To further simplify the analysis we assume also that $\mathrm{L}$ may be taken as a typical horizontal scale characterising not only the horizontal seasonal layer and wind stress variations but also the region of closed $f / h$ contours, $L=O\left(\frac{1}{h} \frac{\partial h}{\partial n}\right)^{-1}=0\left(\frac{\partial}{\partial n}(f / h) . h / f\right)^{-1}$. The integration of Eqs. (3.11) and (3.12) then yields for the barotropic velocity

$$
\hat{\mathrm{u}}=0\left(\frac{\bar{\psi}_{\mathrm{s}}}{\mathrm{hL}}\right)=0\left(\frac{\tau_{\mathrm{s}} \cdot \mathrm{T}_{\mathrm{a}}}{2 \pi \cdot \mathrm{h}}\right)
$$

Compared with the open contour case, Eq. (6.3), we see that the barotropic velocity has been enhanced by the factor $T_{a} / 2 \pi \cdot \beta L \approx 10^{2}$. Thus the seasonal variations of the barotropic velocity are estimated to be of order $0.1 \mathrm{~m} \mathrm{~s}^{-1}$ in regions of closed $\mathrm{f} / \mathrm{h}$ contours, as opposed to $10^{-3} \mathrm{~m} \mathrm{~s}^{-1}$ for open contour regions. The barotropic velocities furthermore lag the wind and thermal forcing by $90^{\circ}$, as opposed to the in-phase relation for open contour regions. The horizontal displacements are similarly almost two orders of magnitude greater than in the open contour case, $\Delta x=0.1 \mathrm{~m} \mathrm{~s}^{-1} \cdot 100$ days $\approx 1,000 \mathrm{~km}$. The vertical displacements, however, remain small, as the resonant trapped vorticity gyre is non-divergent.

The relatively large barotropic velocities, large horizontal displacements and $90^{\circ}$ phase difference between the forcing and barotropic seasonal response represent pronounced signals that should be detectable in regions of closed $f / h$ contours. These regions may therefore be more interesting for a pilot ocean monitoring program than the regions of open $f / h$ contours, in which significant variability on a seasonal time scale is expected only in the seasonal layer, in accordance with traditional concepts.

\section{Summary and Conclusions}

Models of the time dependent ocean circulation can be simplified considerably by filtering out all short term, small scale motions which are unimportant for climatic processes. For time scales large compared with a day and space scales large compared with the internal Rossby radius of deformation $(\sim 50 \mathrm{~km})$, the currents in most of the 
interior ocean can be determined diagnostically as quasi-equilibrium fields, so that only the salinity and temperature fields need be treated prognostically.

Regions of closed $\mathrm{f} / \mathrm{h}$ contours, however, represent exceptions. Here trapped vorticity gyres exist as free flow solutions without external forcing, and in the presence of forcing the barotropic velocity field must therefore be determined prognostically through a potential vorticity equation for the gyres.

Lateral boundary layers and the equatorial regions also require separate treatment. These were not considered specifically, but it is suggested that integrated (parametrical) models analogous in structure to mixed-layer models or the integrated boundary layer models of aerodynamics may be the most appropriate technique for coupling these regions to the interior ocean in a comprehensive ocean model suitable for climate studies.

A coupled multi-region model of the global ocean circulation based on these scale considerations could be sufficiently cost-effective to permit systematic investigation of the role of the oceanic heat storage and transport in climate variability studies over a wide spectrum of space and time scales.

The analysis of the seasonal variations of the interior ocean circulation represents a simple example in which the filtered model yields considerably simpler and more readily interpretable results than a fully three-dimensional, unfiltered model.

\section{$\underline{\text { References }}$}

Bryan, K. (1975): Three-dimensional numerical models of the ocean circulation. Proc. Symposium on Numerical Models of Ocean Circulation, Durham, N.H. National Academy of Sciences, Washington, D.C.

Bryan, K. (1979): Models of the world ocean. Dynamics of Atmospheres and Oceans 3: 327-338.

Bryan, K. and L.J. Lewis (1979): A water mass model of the world ocean. Journal of Geophysical Research 84 C5: 2503-2517.

Ellis, J.S., T.H. Vonder Haar, S. Levitus and A.H. Oort (1978): The annual variation in the global heat balance of the earth. Journal of Geophysical Research 83 C4: 1958-1962.

Frankignoul, C. and K. Hasselmann (1977): Stochastic climate models, Part 2, Application to sea surface temperature anomalies and thermocline variability. Tellus 29: 289305.

GARP (Global Atmospheric Research Programme) (1975): The Physical Basis of Climate and Climate Modelling. Publ. No. 16. WMO, Geneva.

Gill, A.E. and P.P. Niiler (1973): The theory of the seasonal variability in the ocean. Deep-Sea Research 20: 141-177.

Gïnther, H., W. Rosenthal, T.J. Weare, B.A. Worthington, K. Hasselmann and J.A. Ewing (1979): A hybrid parametrical wave prediction model. Journal of Geophysical 
Research 84 C9: 5727-5738.

Hasselmann, K. (1976): Stochastic climate models, Part 1, Theory. Tellus 28: 473-485.

Hasselmann, K., T.P. Barnett, E. Bouws, H. Carlson, D.E. Cartwright, K. Enke, J.A. Ewing, H. Gienapp, D.E. Hasselmann, P. Kruseman, A. Meerburg, P. Müler, D.J. Olbers, K. Richter, W. Sell and H. Walden (1973): Measurements of wind-wave growth and swell decay during the Joint North Sea Wave Project (JONSWAP). Deutsche Hydrographische Zeitschrift, Suppl. A, 8(12).

Holland, W.R. (1967): On the wind-driven circulation in an ocean with bottom topography. Tellus 19: 582-600.

Holland, W.R. (1977): Oceanic general circulation models. In: E.D. Goldberg et al. (Eds.), The Sea: Ideas and Observations on Progress in the Study of the Seas. John Wiley and Sons, Inc., New York.

Holland, W.R. (1979): The general circulation of the ocean and its modeling. Dynamics of Atmospheres and Oceans 3: 111-142.

Kamenkovich, V.M. (1962): On the theory of the Antarctic circular current. Trudy Instituta Okeanologie 56: 241-293.

Kraus, E.B. (Ed.) (1977): Modelling and Prediction of the Upper Layers of the Ocean. Proc. NATO Advanced Study Institute, Pergamon Press, Oxford, 325 pp.

Lemke, P. (1977): Stochastic climate models, Part 3, Application to zonally averaged energy models. Tellus 29: 385-392.

McWilliams, J.C. (1977): A note on a consistent quasi-geostrophic model in a multiply connected domain. Dynamics of Atmospheres and Oceans 1: 427-441.

Oort, A.H. and T.H. Vonder Haar (1976): On the observed annual cycle in the oceanatmosphere heat balance over the northern hemisphere. Journal of Physical Oceanography 6: 781-800.

Pedlosky, J. (1979): Geophysical Fluid Dynamics. Springer, New York-Heidelberg-Berlin.

Pond, S. and K. Bryan (1976): Numerical models of the ocean circulation. Reviews of Geophysics and Space Physics 14: 243-263.

Reynolds, R.W. (1978): Sea surface temperature anomalies in the North Pacific Ocean. Tellus 30: 97-103.

Reynolds, R.W. (1979): A stochastic forcing model of sea surface temperature anomalies in the North Pacific and North Atlantic. Report No. 8, Climatic Research Inst. and Dept. of Atmospheric Sciences, Oregon State University, Corvallis.

Sarkisyan, A.S. and V.F. Ivanov (1971): Joint effect of baroclinicity and bottom relief as an important factor in the dynamics of sea currents. Izvestiya Academy of Sciences, USSR Atmospheric and Oceanic Physics 7: 173-188 (English, 116-124).

Sarkisyan, A.S. and V.P. Keondzhian (1972): Calculation of the surface level and total mass transport function for the North Atlantic. Izvestiya Academy of Sciences, USSR Atmospheric and Oceanic Physics 8: 1202-1215 (English, 701-708). 
Schott, F. and H. Stommel (1978): Beta spirals and absolute velocities in different oceans. Deep-Sea Research 25: 961-1010.

Schulman, E.E. (1975): Numerical models of ocean circulation: a study of topographic effects. Proc. Symposium on Numerical Models of Ocean Circulation, Durham, N.H. National Academy of Sciences, Washington, D.C.

Schulman, E.E. and P.P. Niiler (1979): Topographic effects on the wind-driven ocean circulation. Geophysical Fluid Dynamics 1: 439-462.

Stommel, H. and F. Schott (1977): The beta spiral and the determination of the absolute velocity field from hydrographic station data. Deep-Sea Research 24: 325-329.

Sverdrup, H.U., M.W. Johnson and R.H. Fleming (1942): The Oceans, Their Physics, Chemistry and General Biology. Prentice Hall, Inc., Englewood Cliffs.

Welander, P. (1968): Wind-driven circulation in one and two-layer oceans of variable depth. Tellus 20: 1-15.

Wunsch, C. (1978): The North Atlantic general circulation west of $50^{\circ} \mathrm{W}$ determined by inverse methods. Reviews of Geophysics and Space Physics 16: 583-620. 